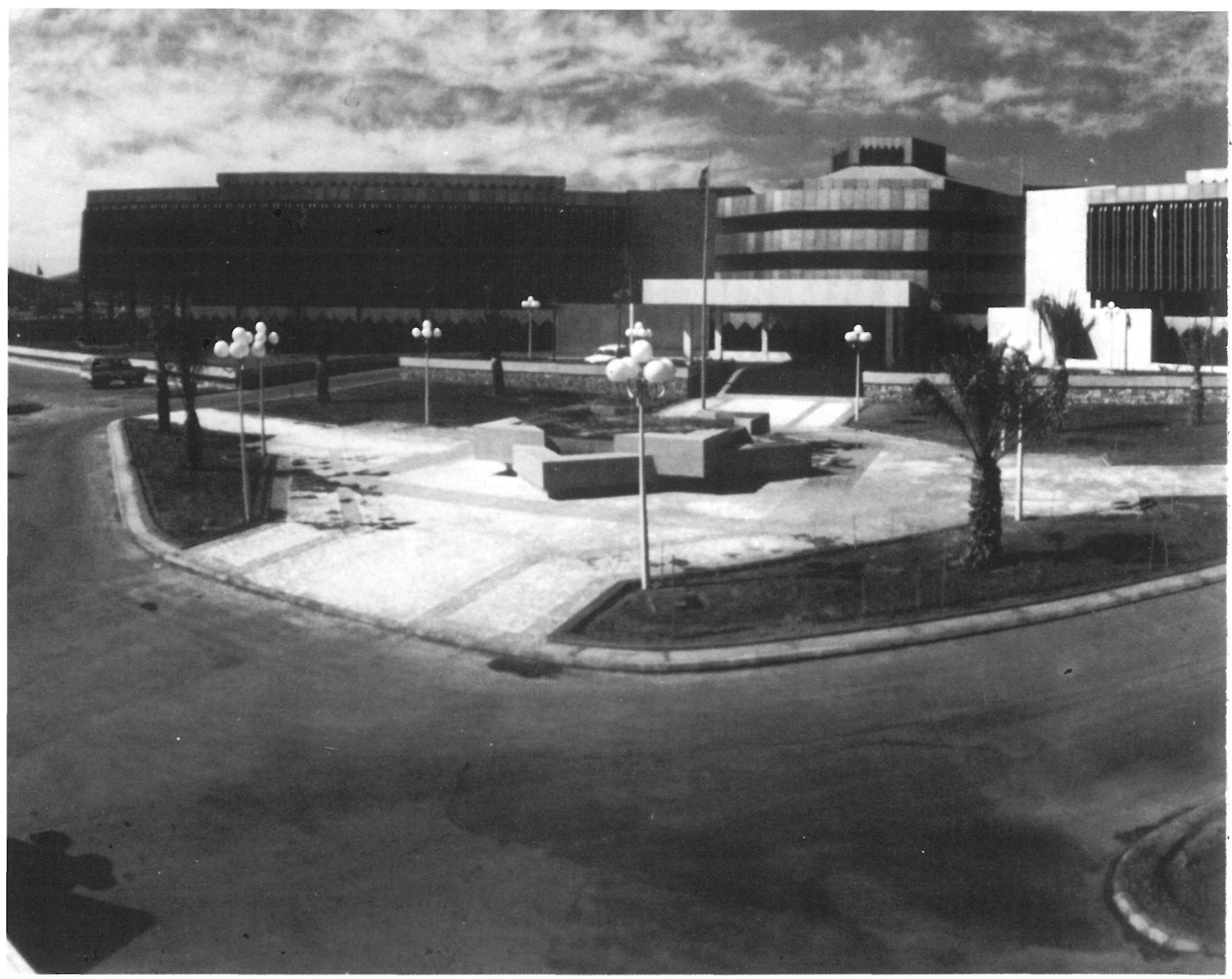

\section{Royal Massarah Hotel Arabia Saudita}

Axe, arquitecto sinopsis

$147-46$

Este edificio ha sido construido en el tiempo récord de ocho meses por OTH International, en asociación con Rashid Engineering. Esta situado a $15 \mathrm{~km}$ de Taifa, en la carretera del aeropuerto.

El Hotel tiene planta en estrella de 5 brazos, con tres niveles de altura. Comprende 120 habitaciones, 30 suites y 5 suites imperiales de $216 \mathrm{~m}^{2}$ cada una.
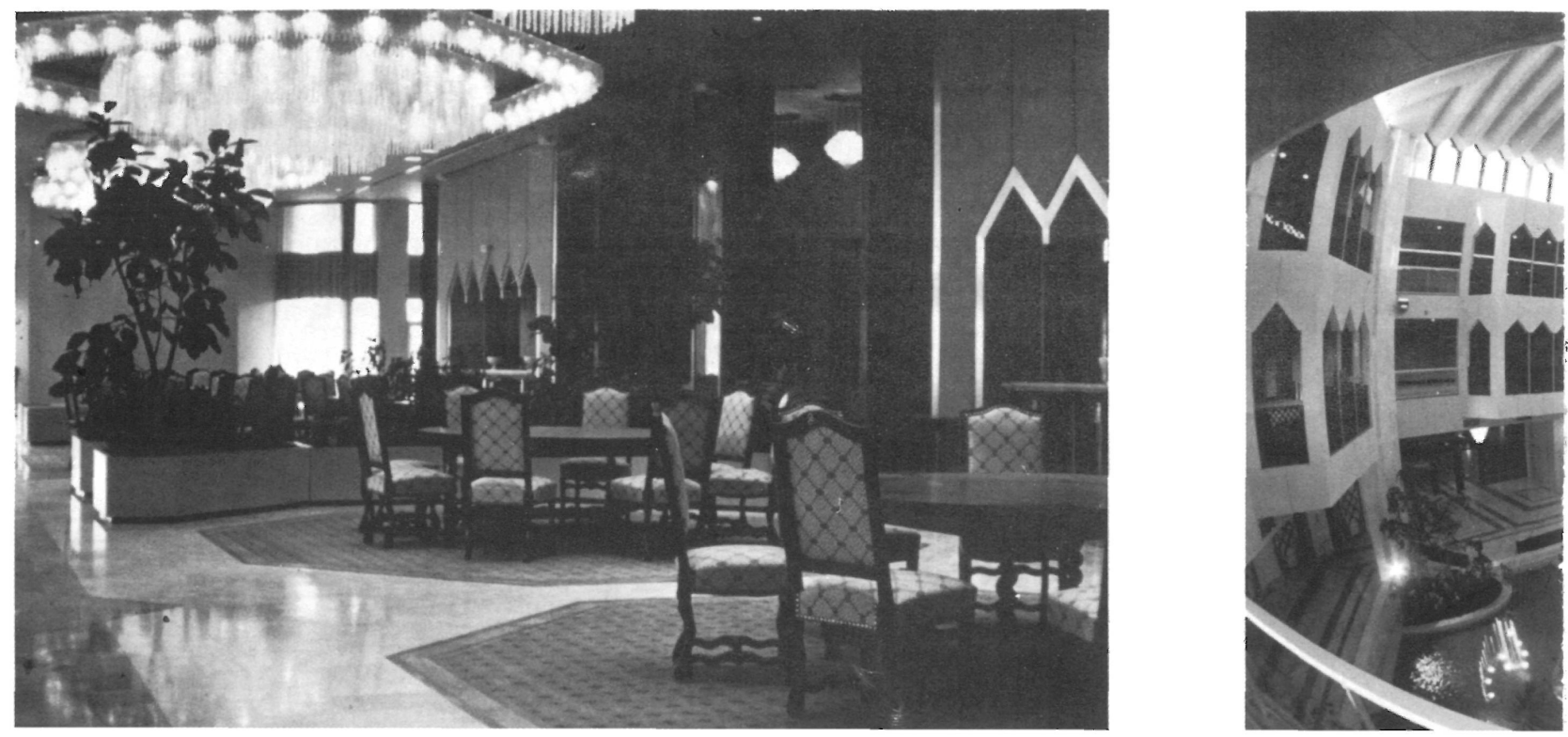


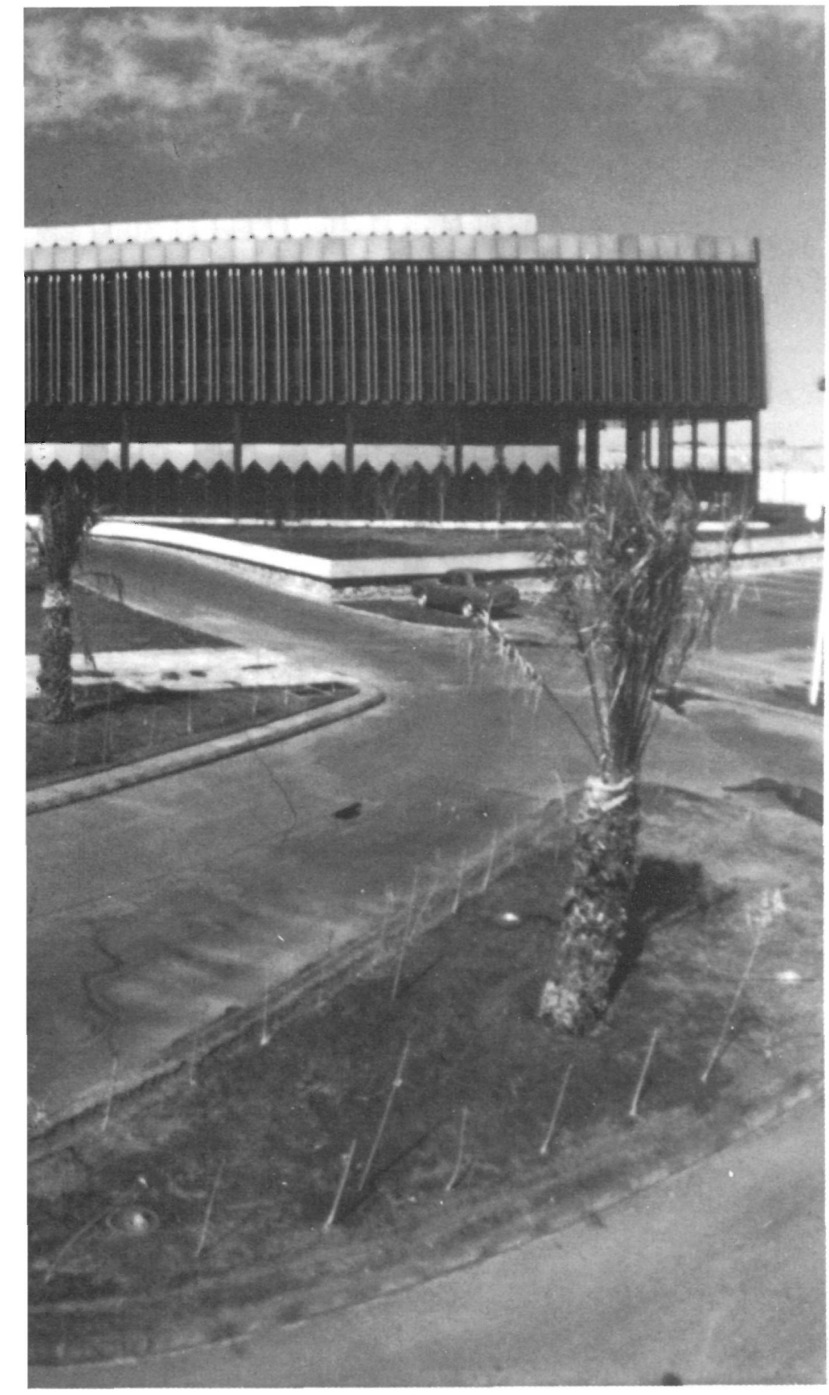

Tanto las instalaciones como el bloque de dirección están separados del cuerpo principal.

La estructura es metálica - sobre retícula de $6 \times 6 \mathrm{~m}-$ y los forjados de hormigón, con cerramientos de muros-cortina partesoles verticales de aluminio.

Dispone de todas las comodidades propias de este tipo de edificios, con lujosa decoración y mobiliario de inspiración en consonancia con el país.

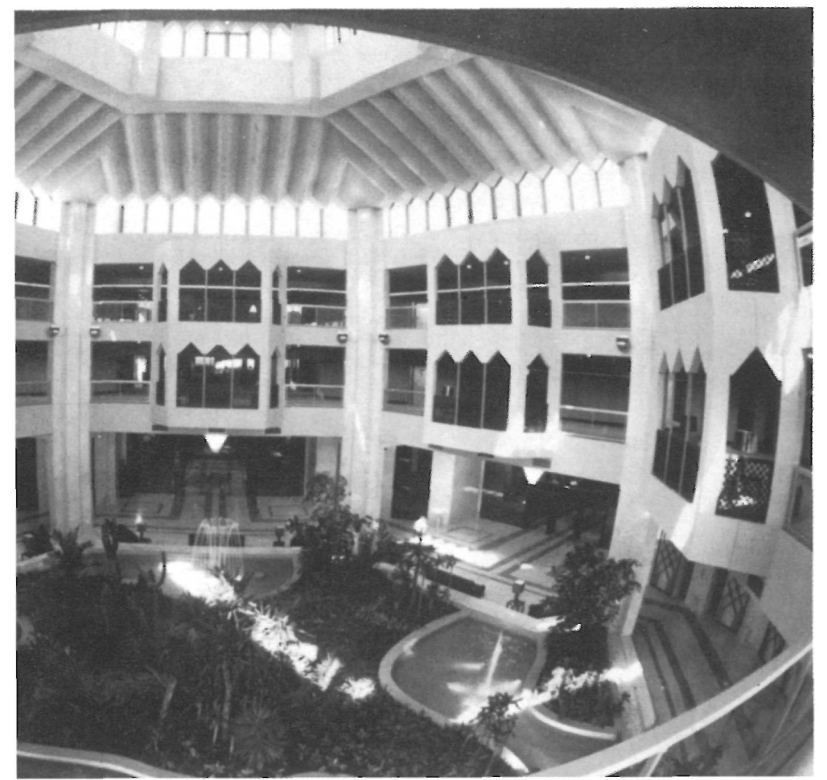

Recientemente, el rey Khaled inauguró el Real Hotel Massarah, en Taifa, que ha sido proyectado y construido en el tiempo récord de ocho meses, por un equipo de proyectos y construcción francés.

OTH Internacional, asociado con Rashid Ingenieros, ha jugado un papel excepcional en ta realización.

Con el fin de llevar a cabo las exigencias establecidas por la propiedad, fue necesario conjuntar al máximo la redacción del proyecto y la coordinación del trabajo de modo que se llegara a la meta de tiempo y acabados previstos.

El edificio consta de cinco alas idénticas, alrededor de un núcleo central, que fueron levantadas simultáneamente en sus tres plantas, altura elegida como más óptima desde el punto de vista de la rapidez elegida.

Los servicios de generadores, aire acondicionado, lavandería, almacenes, entretenimiento y viviendas de dirección se han instalado en edificios separados - en el plazo de tres meses - a fin de que pudieran ser perfectamente utilizados durante la construcción del bloque principal.

La estructura general vertical es metálica, sobre retícula de $6 \times 6 \mathrm{~m}$, placas nervadas, con encofrados metálicos, hormigonados por bombeo y todo ello organizado de tal manera que se favorezcan al máximo la rapidez de ejecución tantas veces mencionados.

Las fachadas son, en general, de muros-cortina, con partesoles de aluminio o de madera.

Tabiquería prefabricada, a base de marcos metálicos y yeso.

Unidades o bloques sanitarios igualmente prefabricados, en cuartos de baño.

Suelos y techo aislados, para aprovechar racionalmente el aire acondicionado y central de refrigeración de $4 \times 600.000$ frigorías/hora.

La planta baja alberga: recepción, vestíbulo, salas de reuniones y banquetes, cafetería, restaurante, tiendas.

La primera se compone de: 120 dormitorios, 30 suites y 5 suites imperiales de $216 \mathrm{~m}^{2}$ cada una con 2 dormitorios, 2 salones, comedor, despacho, cocina y 3 cuartos de baño.

Sótano: cocinas, dormitorios de servicio y de empleados.

OTH Internacional se ha ocupado del proyecto y organización general, preparación de documentos para la construcción, subcontratas, coordinación de suministradores y supervisión técnica. 

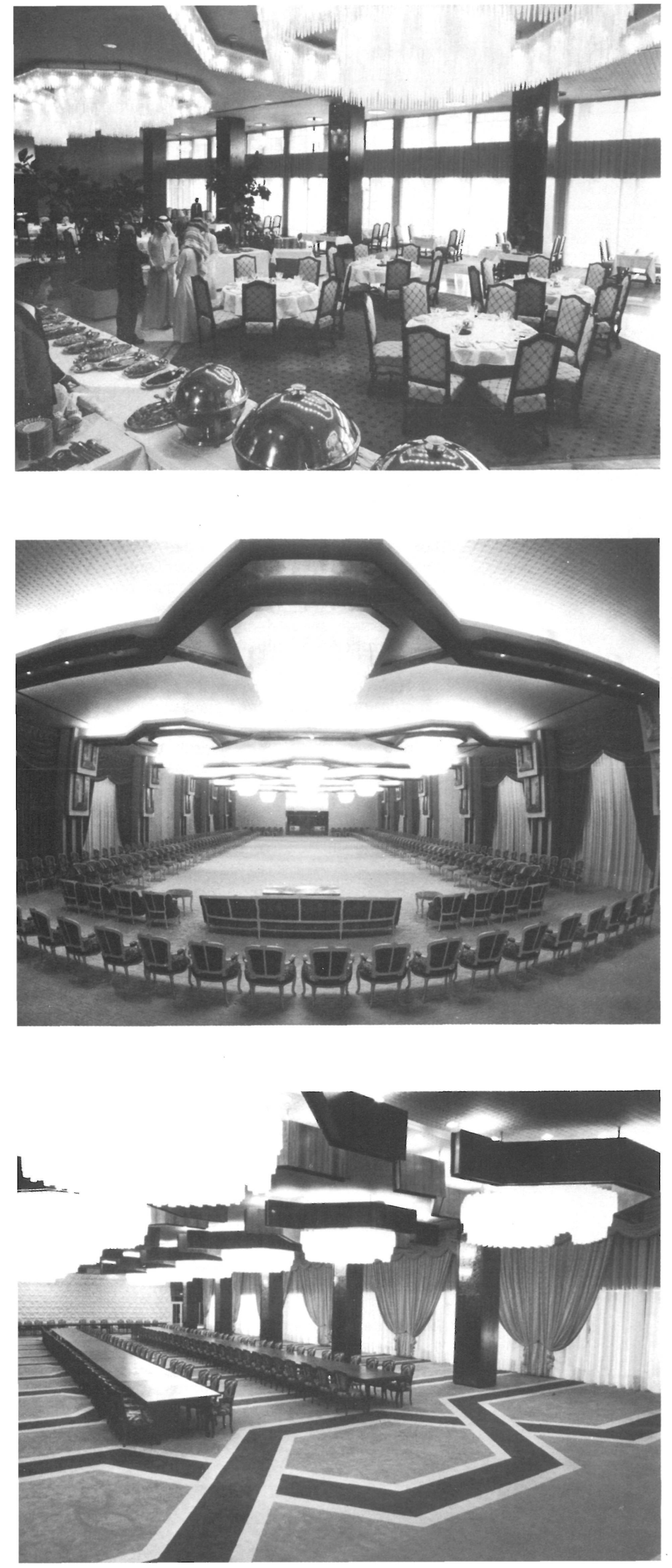

(C) Consejo Superior de Investigaciones Científicas Licencia Creative Commons 3.0 España (by-nc)
Ficha técnica:

Consultores: Rashid Ingenieros

Proyecto:

OTH

Arquitectos:

Axe

Decoración Interior: Inter-Art

Contratista general: Oger

\section{résumé}

ROYAL MASSARAH HOTEL - ARabie Saoudite

Axe, architecte

Cet édifice, situé à 15 kilomètres de Taif sur la route de l'aéroport, a été construit en un temps reccord de huit mois, pa OTH International associé à Rashid Engineering.

Cet hôtel a été édifié sur un plan en étoile de cinq branches et sur trois niveaux de hauteur. II comprend 120 chambres, 30 suites et 5 suites impériales de $216 \mathrm{~m}^{2}$ chacune.

Le bloc de direction et les installations sont séparés du corps principal.

La structure est métallique-sur réticule de $6 \times 6 \mathrm{~m}$-et les hourdis de béton, les cloisons extérieures sont des murs-rideaux avec des brises soleil verticaux d'aluminium. L'hôtel dispose de toutes les commodités propres à ce type d'édifices. La décoration luxueuse et le mobilier sont d'inspirations en consonance avec le pays.

\section{summary}

\section{ROYAL MASSARAH HOTEL}

Saudi Arabia

\section{Axe, Architect}

This building was built in the record time of eight months by OTH International, in conjunction with Rashid Engineering. It is $15 \mathrm{~km}$. from Taif, on the road to the Airport.

The Hotel has a five-point star plan view, with three height levels. It has 120 rooms, 30 suites and 5 imperial suits of $216 \mathrm{~m}^{2}$ each.

Both the installations and the management block are separate from the main body.

The structure is metal - on a $6 \times 6 \mathrm{~m}$. network - and the concrete cladding, with curtain-wall enclosures and vertical aluminium sun protectors.

It has all the conveniences fitting in this type of building, with luxurious decoration and furniture inspired in keeping with the country.

\section{zusammenfassung}

ROYAL MASSARAH HOTEL

\section{Saudi-Arabien}

Axe, Architekt

Dieses Gebäude wurde in der Rekordzeit von acht Monaten durch OTH International in Zusammenarbeit mit Rashid Engineering erstellt. Es liegt, $15 \mathrm{~km}$ von Taif entfernt, an der Strasse des Lufthafens.

Das Hotel hat einen Grundriss in Form eine 5-armigen Sterns und drei Stockwerke. Es verfügt über 120 Zimmer, 30 Suiten und 5 Kaisersuiten von je $216 \mathrm{~m}^{2}$.

Sowohl die Einrichtungen wie auch der Direktionsblock sind vom Hauptkörper getrennt.

Die Struktur ist aus Metall -auf Raster von $6 \times 6 \mathrm{~m}$ - und das Fachwerk aus Beton, mit Abschluss durch Vorhangmauern und mit vertikalen Sonnenschutzteilen aus Aluminium. Es verfügt über den bei derartigen Bauten üblichen Komfort, mit Luxusausstattung und einem dem Landestil entsprechenden Mobiliar. 